\title{
ACTINOMICETOS CON ACTIVIDAD ANTI-CANDIDA AISLADAS DE HORMIGAS CORTADORAS DE HOJAS Atta cephalotes (Formicidae: Myrmicinae: Attini)
}

\author{
Cindy Abigail Gutierrez-Espinoza1,a, Jorge León-Quispe
}

\begin{abstract}
RESUMEN
Objetivos. Aislar, seleccionar e identificar actinomicetos asociados a hormigas cortadoras de hojas Atta cephalotes (Linnaeus, 1758), que presenten mayor actividad anti-Candida. Materiales y métodos. Estudio transversal realizado en hormigas recolectadas de una localidad de Huánuco, Perú, a partir de las cuales se aislaron cepas de actinomicetos que fueron evaluadas mediante pruebas in vitro para determinar su capacidad antagonista frente a especies de Candida. Los actinomicetos de mayor antagonismo fueron seleccionados y cultivados en agitación, luego se obtuvieron los metabolitos extracelulares con solventes orgánicos y finalmente se evaluaron los extractos crudos para determinar cuantitativamente la concentración mínima inhibitoria (CMI). Resultados. Se logró aislar 30 actinomicetos, de los cuales el $47 \%$ presentaron actividad antagonista a Candida albicans (C. albicans) ATCC 7516, el $43 \%$ a Candida parapsilosis ATCC 7307, el 37\% a Candida tropicalis ATCC 7206 y C. albicans ATCC 10231 y el 30\% a C. albicans ATCC 98028. Extractos orgánicos de las cepas HAA-16 y HAA-17 presentaron marcada actividad anti-Candida; siendo el extracto de acetato de etilo de la cepa HAA17 el de mejor rendimiento por tener mayor espectro de actividad y presentar una CMI de $3,25 \mathrm{mg} / \mathrm{ml}$ frente a $C$. albicans ATCC 7516 y Candida parapsilosis ATCC 7307. Los actinomicetos seleccionados se identificaron mediante técnicas moleculares como miembros del género Streptomyces. Conclusiones. Los actinomicetos asociados a Atta cephalotes son excelentes productores de compuestos bioactivos, capaces de inhibir el crecimiento de levaduras patógenas del género Candida y con potencial aplicación en el desarrollo de nuevos productos naturales de interés biomédico.
\end{abstract}

Palabras claves: Productos naturales; Actinobacterias; Antagonista; Levaduras; Streptomyces (Fuente: DeCS BIREME)

\section{ACTINOMYCES WITH ANTI-CANDIDA ACTIVITY ISOLATED FROM LEAF-CUTTING ANTS ATTA CEPHALOTES (FORMICIDAE: MYRMICINAE: ATTINI)}

ABSTRACT

Objectives. To isolate, select and identify actinomyces associated to leaf-cutting ants Atta cephalotes (Linnaeus, 1758), that present a greater anti-Candida activity. Materials and Methods. Cross-sectional study made with ants collected at a location in Huánuco, Peru, from which strands of actinomyces were isolated and later evaluated by in vitro testing in order to determine its antagonistic capacity against species of Candida. The actinomyces with greater antagonism were selected and cultured by agitation, then the reliable extracellular metabolites were obtained with organic solvents, and finally the crude extracts were evaluated to determine quantitatively the minimum inhibiting concentration (MIC). Results. Thirty (30) actinomyces were isolated, of which $47 \%$ exhibited antagonistic activity against Candida albicans (C. albicans) ATCC 7516, 43\% to Candida parapsilosis ATCC 7307, 37\% to Candida tropicalis ATCC 7206 and C. albicans ATCC 10231, and 30\% to C. albicans ATCC 98028. Organic extracts of the HAA-16 and HAA-17 strands exhibited noticeable anti-Candida activity, being the ethyl acetate extract of the HAA-17 strand the one with the highest performance thanks to a wider activity spectrum of MIC $3.25 \mathrm{mg} / \mathrm{mL}$ against C. albicans ATCC 7516 and Candida parapsilosis ATCC 7307. The selected actinomyces were identified by means of molecular techniques as members of the Streptomyces genus. Conclusions. Actinomyces associated to Atta cephalotes are excellent producers of bioactive compounds, being able to inhibit the growth of pathogenic mold of the Candida genus and with potential for application in the development of new natural products for the biomedical field.

Keywords: Natural products; Actinobacteria; Antagonist; Yeasts, Streptomyces (source: MeSH NLM).

Este estudio forma parte de la tesis titulada «Aislamiento, caracterización y evaluación de la capacidad antimicrobiana de actinomicetos asociados a hormigas cortadoras de hojas (Formicidae: Myrmicinae: Attini)» perteneciente a la autora Cindy Abigail Gutierrez Espinoza para optar al título de bióloga con mención en Zoología en la Universidad Nacional Mayor de San Marcos. Lima, Perú

1 Laboratorio de Ecología Microbiana, Facultad de Ciencias Biológicas, Universidad Nacional Mayor de San Marcos, Lima, Perú.

a Biólogo; ${ }^{\mathrm{b}}$ magíster en Ciencias Microbiológicas

Recibido: 16/05/2018 Aprobado: 26/09/2018 En línea: 21/12/2018

Citar como: Gutierrez-Espinoza CA, León-Quispe J. Actinomicetos con actividad anti- Candida aisladas de hormigas cortadoras de hojas Atta cephalotes (Formicidae: Myrmicinae: Attini). Rev Peru Med Exp Salud Publica. 2018;35(4):590-8. doi:10.17843/rpmesp.2018.354.3673. 


\section{INTRODUCCION}

Las infecciones fúngicas se han incrementado en frecuencia e importancia en las últimas décadas, acompañadas de una alta mortalidad, generalmente ocasionadas por infecciones del torrente sanguíneo ${ }^{(1)}$. La candidiasis es una micosis oportunista causada por diferentes especies de levaduras del género Candida, siendo la especie más importante Candida albicans (C. albicans) por presentarse en el $80 \%$ de las infecciones ${ }^{(2)}$. A pesar de que la sensibilidad a los antifúngicos, principalmente al fluconazol, es alta para $C$. albicans, otras especies están aumentando significativamente su resistencia y muchas de ellas presentan baja sensibilidad a los antifúngicos utilizados tradicionalmente, especialmente las especies Candida krusei y Candida glabrata ${ }^{(3)}$. Este problema de resistencia obliga a la comunidad cientíica a identificar nuevos antimicrobianos o de diseñar inhibidores de enzimas que confieran resistencia a antibióticos ${ }^{(4)}$.

El descubrimiento de nuevos compuestos antimicrobianos y de otros metabolitos secundarios bioactivos requiere la búsqueda de nuevas especies o géneros de microorganismos productores de estos metabolitos, pero en ambientes menos explorados ${ }^{(5)}$. Los actinomicetos son bacterias filamentosas grampositivas, con alto contenido de guanina y citosina (G-C)en suADN y biotecnológicamente importantes, puesto que son útiles para la producción de metabolitos secundarios, especialmente los antibióticos, antifúngicos y/o antitumorales ${ }^{(6)}$. Aproximadamente 23 000 metabolitos secundarios con actividad biológica son producidos por microorganismos y más de 10000 de estos compuestos son producidos por actinomicetos; de ahí la importancia de seguir investigando los actinomicetos por su gran capacidad para producir compuestos bioactivos ${ }^{(7)}$. La mayoría de los actinomicetos son comúnmente aislados del suelo; sin embargo, después de décadas de bioprospección de este entorno, cada vez es más difícil obtener cepas que produzcan nuevos metabolitos bioactivos ${ }^{(8)}$.

Por otro lado, el descubrimiento de nuevos actinomicetos provenientes de ecosistemas no estudiados parece ser una de las fuentes más interesantes y prometedoras de nuevos compuestos biológicamente activos ${ }^{(9)}$. El papel de los actinomicetos en las relaciones simbióticas con artrópodos ha sido recientemente estudiado, siendo uno de los sistemas naturales más caracterizados el de los actinomicetos asociados a las hormigas cortadoras de hojas (Hymenoptera: Formicidae: Attini) ${ }^{(10,11)}$. Este sistema es un mutualismo obligado, donde las hormigas para sobrevivir dependen de su simbionte (hongos, actinomicetos u otros); y como consecuencia de ello, las hormigas protegen al simbionte de amenazas, ya sea de otras bacterias o de parásitos especializados como el hongo necrotrófico del género Escovopsis sp. ${ }^{(12)}$. La estrategia de defensa de las hormigas cortadoras de hojas ocurre por mecanismos químicos o por actinomicetos asociados a su

\section{MENSAJES CLAVE}

Motivación para realizar el estudio. El incremento incesante de la resistencia de microorganismos patógenos hacia los antimicrobianos es una seria amenaza a la Salud Pública. Este hecho motiva la necesidad de explorar nuevas fuentes para buscar microorganismos productores de antimicrobianos novedosos.

Principales hallazgos. Se aislaron 30 cepas de actinomicetos asociadas a Atta cephalotes, donde 16 cepas presentaron actividad, al menos, a una cepa del género Candida.

Implicancias. Los actinomicetos asociados a hormigas cortadoras de hojas contribuirán en la producción de nuevos metabolitos que podrían controlar la resistencia antimicrobiana.

cutícula, principalmente de los géneros Pseudonocardia y Streptomyces ${ }^{(13)}$.

Basados en experiencias previas, esta investigación se llevó a cabo con el objetivo de aislar, seleccionar e identificar cepas de actinomicetos asociados al organismo Atta cephalotes (Linnaeus, 1758) que presenten mayor actividad anti-Candida. Nuestros resultados permitirán en el futuro diseñar estrategias de producción y desarrollo de nuevos productos naturales basados en metabolitos microbianos con principios bioactivos eficaces en el control de patógenos emergentes.

\section{MATERIALES Y MÉTODOS}

\section{DISEÑO DE ESTUDIO}

Se realizó un estudio transversal en hormigas cortadoras de hojas de la especie Atta cephalotes colectadas durante el mes de enero del 2015 en una localidad del distrito Mariano Dámaso Beraún ( $9^{\circ} 21^{\prime} 22^{\prime \prime} \mathrm{S}, 75^{\circ} 58^{\prime} 27^{\prime \prime}$ O) en la provincia de Leoncio Prado, Departamento de Huánuco en Perú.

\section{COLECTA DE HORMIGAS}

La colecta se llevó a cabo en un solo muestreo, de forma manual y en condiciones asépticas. Las hormigas se almacenaron en tubos estériles y fueron transportadas en cajas refrigeradas $4^{\circ} \mathrm{C}$ hacia el Laboratorio de Ecología Microbiana de la Facultad de Ciencias Biológicas de la Universidad Nacional Mayor de San Marcos para su procesamiento.

\section{AISLAMIENTO DE ACTINOMICETOS ASOCIADOS A Atta cephalotes}

Previo al aislamiento, las hormigas fueron liberadas de cualquier partícula externa. El aislamiento de los actinomicetos se realizó a partir de $10 \mathrm{~g}$ de hormigas, las cuales fueron 
sumergidas en $90 \mathrm{~mL}$ de solución salina estéril $\left(10^{-1}\right)$ y homogenizadas enérgicamente en un vórtex. La dilución fue sometida a shock térmico; luego se realizaron diluciones seriadas hasta $10^{-4}$. Se tomó $100 \mu \mathrm{L}$ de cada dilución y se sembraron en los medios Agar Almidón Caseína (AAC) y Agar Czapek-Dox (ACZ), suplementados con cicloheximida $\left(100 \mu \mathrm{g} \cdot \mathrm{mL}^{-1}\right)$ y ácido nalidíxico $\left(25 \mu \mathrm{g} \cdot \mathrm{mL}^{-1}\right)^{(14)}$. Los cultivos fueron incubados a $28^{\circ} \mathrm{C}$ por 15 días; al cabo de este tiempo se seleccionaron colonias típicas de actinomicetos, los cuales previa caracterización macroscópica y microscópica se conservaron en ceparios de AAC más glicerina $(20 \% \mathrm{v} / \mathrm{v})$ para estudios posteriores ${ }^{(15)}$.

\section{DETERMINACIÓN DE LAACTIVIDAD ANTI-CANDIDA}

\section{Cepas indicadoras}

Las cepas testigo utilizadas en el presente estudio pertenecen a levaduras del género Candida $(C)$ : $C$. albicans ATCC 10231, C. albicans ATCC 7516, C. albicans ATCC 98028, C. tropicalis ATCC 7206 y C. parapsilosis ATCC 7307 , las cuales forman parte de la colección del Laboratorio de Ecología Microbiana de la Universidad Nacional Mayor de San Marcos.

\section{Actividad anti-Candida}

Se realizó mediante el método de «doble capa» según León et al. (2007) ${ }^{(15)}$. Los actinomicetos fueron sembrados como macrocolonias en AAC e incubados a $28^{\circ} \mathrm{C}$ por $5-7$ días. Las cepas de Candida fueron reactivadas en caldo Sabouraud por 24-48 h hasta alcanzar la escala 0,5 de Mac Farland. Las pruebas de antagonismo se realizaron mediante la inoculación de las cepas testigos en medio semisólido licuado y agregado a modo de segunda capa sobre los cultivos de los actinomicetos. Los cultivos fueron incubados a $30^{\circ} \mathrm{C}$ por $24-48 \mathrm{~h}$. Posteriormente, se realizaron las lecturas correspondientes, observando y midiendo los halos de inhibición (en $\mathrm{mm}$ de diámetro). Las pruebas se realizaron por triplicado. El cálculo del porcentaje de inhibición (I) se realizó mediante la siguiente fórmula:

$$
\mathrm{I}=\frac{R 1-R 2}{R 1} \times 100
$$

Donde, $\mathrm{R} 1$ = media del diámetro del halo de inhibición; R2 = media del diámetro de la colonia de actinomiceto. Este ensayo permitió seleccionar a los actinomicetos con mayores halos de inhibición anti-Candida para ensayos posteriores.

\section{OBTENCIÓN DE EXTRACTOS CRUDOS}

Los actinomicetos seleccionados por su mayor actividad anti-Candida se procesaron según las pautas señaladas por Aponte et al. (2016) ${ }^{(16)}$. Se inocularon en Erlenmeyer con $200 \mathrm{~mL}$ del caldo Peptona-Extracto de levadura, incubados a $28{ }^{\circ} \mathrm{C}$ por 10 días en agitación constante de $250 \mathrm{rpm}$. Transcurrido el tiempo, los cultivos fueron centrifugados a $4000 \mathrm{rpm}$ por $25 \mathrm{~min}$ para obtener el sobrenadante libre de células. Para concentrar los metabolitos extracelulares de los cultivos se añadieron solventes como acetato de etilo, diclorometano (polares) y hexano (apolar) en la proporción $1: 1(\mathrm{v} / \mathrm{v})$, luego fueron agitados vigorosamente durante $24 \mathrm{~h}^{(17)}$. Transcurrido el tiempo, los extractos crudos fueron concentrados a presión reducida en un rotavapor $(\mathrm{BUCHI}$ $\mathrm{R}-3000)$ a $40^{\circ} \mathrm{C}$ hasta obtener los residuos secos.

\section{DETERMINACIÓN DE LA ACTIVIDAD ANTI-CANDIDA DE LOS EXTRACTOS CRUDOS}

Se realizó según Pandey et al. (2004) ${ }^{(18)}$. Los residuos secos fueron pesados y resuspendidos en Dimetilsulfóxido (DMSO) al $5 \%$ para obtener las concentraciones iniciales de los extractos orgánicos (mg. $\left.\mathrm{mL}^{-1}\right)$. A partir de estas muestras, $45 \mu \mathrm{L}$ fueron colocadas en pocillos $(5 \mathrm{~mm}$ de diámetro) preparados en Agar Mueller Hinton y sembradas con la cepa testigo. Los cultivos fueron incubados a $30^{\circ} \mathrm{C}$ por 24-48 h. Luego de este tiempo, la zona de inhibición fue medida y registrada (en $\mathrm{mm}$ de diámetro). Las pruebas se realizaron por triplicado.

\section{CONCENTRACIÓN MÍNIMA INHIBITORIA (CMI) DE LOS EXTRACTOS CRUDOS}

Para esta prueba se utilizó como cepas testigo cultivos de $C$. albicans ATCC 10231, C. albicans ATCC 7516, C. albicans ATCC 98028, C. tropicalis ATCC 7206 y C. parapsilosis ATCC 7307 mantenidos en caldo Tripticasa de Soya (TSB) a $30{ }^{\circ} \mathrm{C}$ de incubación. Se realizó siguiendo la metodología de Karthy et al. (2009) (19). Los extractos orgánicos con acetato de etilo de las cepas seleccionadas se disolvieron en DMSO al $5 \%$ para obtener una solución stock, a partir del cual se prepararon diluciones consecutivas (1:2), aplicando $10 \mu \mathrm{L}$ de cada uno en los pocillos de microdilución (Microplacas Pure Grade; Brand); luego, se agregó $80 \mu \mathrm{L}$ de caldo TSB y previa homogenización, se agregó $10 \mu \mathrm{L}$ de las suspensiones de las cepas testigo. Las microplacas fueron incubadas a $30{ }^{\circ} \mathrm{C}$ por $18-24 \mathrm{~h}$. Transcurrido el tiempo se vertió $40 \mu \mathrm{L}$ de cloruro de trifenil tetrazolio (TTC; $5 \mathrm{mg} \cdot \mathrm{mL}^{-1}$ ) en cada pocillo, incubando a $30^{\circ} \mathrm{C}$ por 20 min adicionales. El viraje a rojo indicó crecimiento microbiano. Se consideró como valor de CMl a la menor concentración en que no hubo crecimiento.

\section{IDENTIFICACIÓN GENOTÍPICA}

Los actinomicetos seleccionados fueron cultivados en TSB e incubados a $28{ }^{\circ} \mathrm{C}$ por cinco días. Transcurrido el tiempo, fueron centrifugados a $4000 \mathrm{rpm}$ por $10 \mathrm{~min}$ y el ADN genómico se extrajo según las indicaciones del kit comercial Vivantis $® G F-1$. Mediante reacción en cadena de polimerasa (PCR) se amplificó el gen que codifica ARNr 16S, utilizando los primers universales 27F (5'-AGA GTT TGA TCC TGG CTC AG-3’) y 1492R (5`-TAC GGT TAC CTT GTT ACG ACTT-3'). Los productos de la PCR se secuenciaron mediante los servicios de Macrogen Inc. (Seúl, Corea). Las secuencias se analizaron y editaron mediante el programa BioEdit Sequence Alignment Editor 
7.0.9 y luego se compararon con las secuencias de la base de datos del National Center for Biotechnology Information (NCBI) mediante el algoritmo BLAST (Basic Local Alignment Search Tool) ${ }^{(20)}$. El árbol filogenético se diseñó mediante el programa MEGA 7 de acuerdo con el método estadístico Neighbor-Joining, utilizando el modelo de sustitución Kimura-2-parámetros y con un valor de bootstrap de 1000 réplicas ${ }^{(21)}$.

\section{ANÁLISIS ESTADÍSTICOS}

El criterio utilizado fueron las medidas de los halos de inhibición sobre el crecimiento de la cepa testigo como prueba positiva de actividad anti-Candida mediante un análisis de varianza (ANOVA) de dos vías sin interacción, verificándose los supuestos de normalidad y homogeneidad de varianzas. La significancia se reportó con un nivel de confianza del $95 \%(\alpha=0,05)$. Para realizar estos cálculos, se empleó el programa estadístico INFOSTAT 2018.

\section{RESULTADOS}

\section{AISLAMIENTO DE ACTINOMICETOS Y CARACTERISTIICAS DE LAS COLONIAS}

Se logró aislar un total de 30 cepas de actinomicetos asociados a Atta cephalotes. Del los cuales, 24 (80 \%) se recuperaron en AAC y sólo 6 (20 \%) en ACZ. El tamaño de las colonias fue variable, desde $4 \mathrm{~mm}$ hasta $15 \mathrm{~mm}$ de diámetro; los morfotipos predominantes fueron colonias de color blanco-grisáceas, bordes irregulares, elevadas o planas, convexa y de consistencia dura (Figura 1A). Las observaciones microscópicas mediante la tinción gram mostraron filamentos ramificados grampositivos delgados y largos (Figura 1B), mientras que por técnicas de microcultivo se observaron esporas tanto individuales como las originadas de las hifas ramificadas no fragmentadas, organizadas en forma de cadenas largas y enrolladas en espiral; siendo esta última característica muy propia del género Streptomyces (Figura 1C).

\section{DETERMINACIÓN DE LA ACTIVIDAD ANTI-CANDIDA}

De la totalidad de actinomicetos aislados y evaluados por su actividad anti-Candida, 16 cepas (53\% del total) presentaron actividad al menos frente a una cepa del género Candida; de ellas, 14 (87,5\%) presentaron actividad frente a C. albicans ATCC 7516, $13(81,2 \%)$ frente a C. parapsilosis ATCC 7307, 11 (68,7\%) frente a C. tropicalis ATCC 7206 y C. albicans ATCC 10231 y 10 (62,5\%) a C. albicans ATCC 98028. Los actinomicetos indicados como HAA-16 y HAA17 fueron los más relevantes y seleccionados para estudios complementarios por ser las cepas más efectivas contra $C$. albicans ATCC 10231 con 86,2 \% y 87,1 \% de inhibición, respectivamente (Tabla 1 ). Se verificaron los supuestos

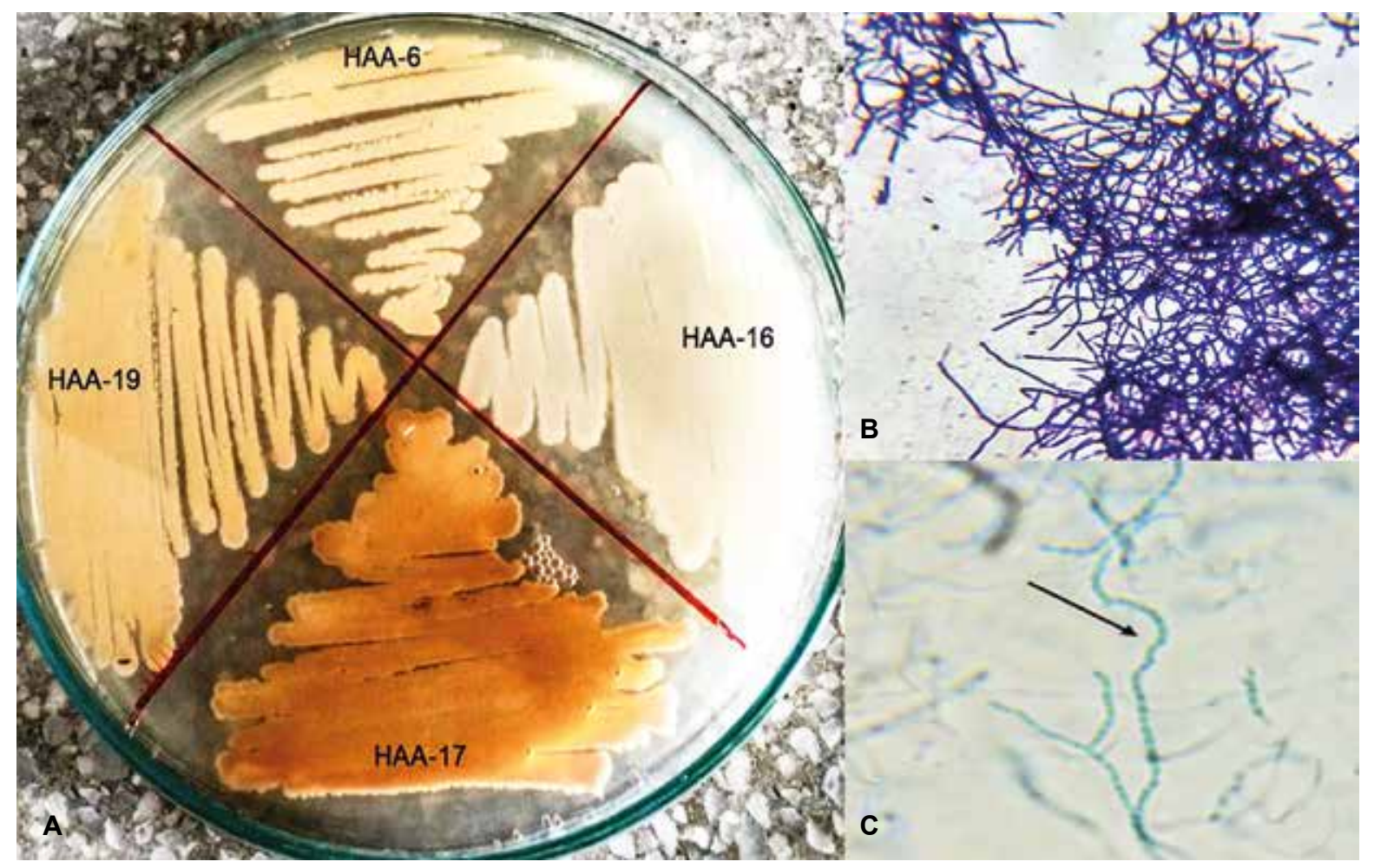

Figura 1. Características macroscópicas de algunos actinomicetos aislados de $A$. cephalotes. A) Crecimiento en agar Almidón Caseína, luego de una incubación de 15 días a $28^{\circ} \mathrm{C}$. B) Coloración gram del actinomiceto HAA-17, hifas en formación a partir de un cultivo de siete días. Aumento 100X. C) Microcultivo de siete días (tinción con Azul de Lactofenol) de la cepa HAA-17, se observan las esporas organizadas en formas de cadenas largas y onduladas (flecha). Aumento 100X. Todas son características del género Streptomyces. 
Tabla 1. Actividad anti-Candida de los actinomicetos activos aislados de A. cephalotes (método de doble capa)

\begin{tabular}{|c|c|c|c|c|c|}
\hline \multirow{2}{*}{$\begin{array}{l}\text { Actinomicetos } \\
(n=16)\end{array}$} & \multicolumn{5}{|c|}{ Cepa indicadora } \\
\hline & $\begin{array}{l}\text { C. tropicalis } \\
\text { ATCC } 7206\end{array}$ & $\begin{array}{c}\text { C. albicans ATCC } \\
98028\end{array}$ & $\begin{array}{l}\text { C. albicans } \\
\text { ATCC } 7516\end{array}$ & $\begin{array}{l}\text { C. parapsilosis } \\
\text { ATCC } 7307\end{array}$ & $\begin{array}{l}\text { C. albicans } \\
\text { ATCC } 10231\end{array}$ \\
\hline HAA-1 & $78,5 \pm 0,2$ & - & $83,2 \pm 0,1$ & $80,2 \pm 0,1$ & - \\
\hline HAA-4 & - & $70,4 \pm 0,1$ & $82,1 \pm 0,1$ & - & $74,2 \pm 0,1$ \\
\hline HAA-6 & $27,3 \pm 0,1$ & - & - & $79,6 \pm 0,1$ & - \\
\hline HAA-7 & $58,2 \pm 0,1$ & - & - & - & - \\
\hline HAA-9 & - & - & $64,9 \pm 0,1$ & $66,7 \pm 0,2$ & $76,3 \pm 0,1$ \\
\hline HAA-11 & - & $60,2 \pm 0,2$ & $62,8 \pm 0,1$ & $63,4 \pm 0,1$ & $76,8 \pm 0,3$ \\
\hline HAA-16 & $79,1 \pm 0,1$ & $40,5 \pm 0,1$ & $74,1 \pm 0,1$ & - & $86,2 \pm 0,1$ \\
\hline HAA-17 & $72,3 \pm 0,3$ & $66,3 \pm 0,1$ & $75,2 \pm 0,2$ & $37,2 \pm 0,3$ & $87,1 \pm 0,1$ \\
\hline HAA-18 & - & - & $77,2 \pm 0,1$ & $66,2 \pm 0,1$ & - \\
\hline HAA-19 & $81,6 \pm 0,1$ & $79,3 \pm 0,1$ & $78,4 \pm 0,1$ & $70,7 \pm 0,1$ & $68,3 \pm 0,2$ \\
\hline HAA-21 & $70,3 \pm 0,2$ & $79,5 \pm 0,3$ & $76,2 \pm 0,1$ & $71,5 \pm 0,1$ & $82,3 \pm 0,1$ \\
\hline HAA-22 & - & $35,4 \pm 0,3$ & $75,3 \pm 0,1$ & $38,6 \pm 0,2$ & $74,7 \pm 0,1$ \\
\hline HAA-23 & $77,7 \pm 0,1$ & $70,8 \pm 0,1$ & $78,6 \pm 0,1$ & $80,4 \pm 0,1$ & $79,3 \pm 0,1$ \\
\hline HAA-25 & $74,5 \pm 0,2$ & - & $79,4 \pm 0,1$ & $80,2 \pm 0,1$ & - \\
\hline HАA-26 & $77,4 \pm 0,1$ & $79,1 \pm 0,1$ & $67,3 \pm 0,2$ & $71,6 \pm 0,1$ & $67,6 \pm 0,2$ \\
\hline HAA-27 & $65,3 \pm 0,3$ & $38,6 \pm 0,2$ & $82,4 \pm 0,1$ & $46,7 \pm 0,3$ & $70,4 \pm 0,1$ \\
\hline
\end{tabular}

Para todos los valores en las celdas la actividad anti-Candida se expresa como la media del porcentaje de inhibición y la desviación estándar Guión (-) : no se encontró actividad anti-candida

de normalidad y homogeneidad de varianzas, no se encontraron diferencias significativas entre los tratamientos $(p=0,134)$.

Se observa el comportamiento de las medias y el error estándar de los porcentajes de inhibición de los actinomicetos frente a las levaduras evaluadas y al contrastar las medias se puede observar ciertas diferencias que no son significativas (Prueba LSD de Fisher) (Figura 2). Entre los otros actinomicetos son destacables las cepas HAA-17, HAA-19,
HAA-21, HAA-23, HAA-26 y HAA-27 por presentar actividad frente a las cinco cepas del género Candida. Asimismo, las pruebas de caracterización fenotípica señalan que todas corresponden al género Streptomyces sp.

\section{DETERMINACIÓN DE LA ACTIVIDAD ANTI-CANDIDA DE LOS EXTRACTOS CRUDOS}

Los extractos de los actinomicetos seleccionados (HAA16 y HAA-17) presentaron actividad frente a las levaduras evaluadas, siendo en su mayoría los extractos con acetato

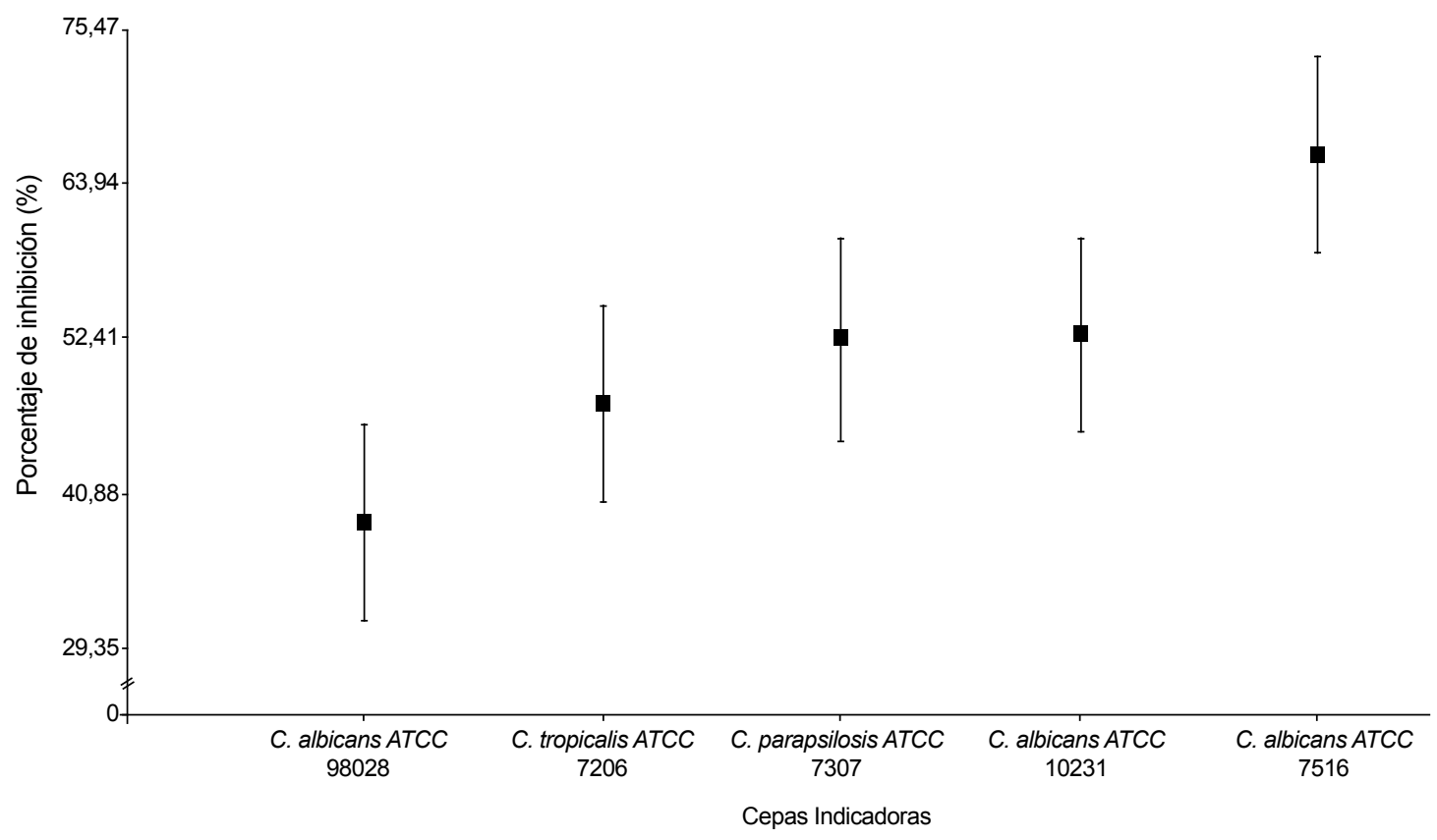

Figura 2. Porcentajes de inhibición de los actinomicetos aislados frente al género Candida. Cada barra representa la media y el error estándar de tres réplicas independientes $(p>0,05)$ 
de etilo los que mostraron mayores halos de inhibición. Es destacable la actividad del extracto de la cepa HAA-17 frente a C. parapsilosis ATCC 7307 con halos de inhibición que alcanzó hasta $30 \mathrm{~mm}$ de diámetro (equivalente a $86 \%$ de inhibición), valor muy cercano al del control positivo Fluconazol $\left(20 \mu \mathrm{g} \cdot \mathrm{mL}^{-1}\right)$ (33 mm de diámetro, $87 \%$ de inhibición) (Figura 3A), mientras que frente a $C$. albicans ATCC 10231 alcanzó halos de hasta $27 \mathrm{~mm}$ de diámetro (equivalente a $85 \%$ de inhibición) (Figura 3B). Por su parte, los extractos diclorometánicos también presentaron resultados favorables para algunas especies, por ejemplo, el extracto de la cepa HAA-16 presentó mayor actividad de inhibición (22 $\mathrm{mm}$ de diámetro) frente a $C$. tropicalis ATCC 7206 en comparación con la del extracto con acetato de etilo (15 mm de diámetro). En cambio, los extractos hexánicos mostraron tener limitada actividad anti-Candida. De acuerdo con estos resultados, el extracto orgánico obtenido con acetato de etilo fue seleccionado para la determinación de la CMI.

\section{CONCENTRACIÓN MÍNIMA INHIBITORIA}

Las concentraciones iniciales de los extractos obtenidos con acetato de etilo de las cepas HAA-16 y HAA-17 fueron en ambos casos de $6,5 \mathrm{mg} \cdot \mathrm{mL}^{-1}$. La CMl de los dos actinomicetos en estudio frente a especies del género Candida varió entre 3,25 - 6,50 mg.mL $\mathrm{mL}^{-1}$. Es destacable la actividad de la cepa HAA-17 frente a C. albicans ATCC 7516 y C. parapsilosis ATCC 7307, donde el extracto logra alcanzar la CMl de 3,25 mg. $\mathrm{mL}^{-1}$ en ambos casos (Figura 4).

\section{IDENTIFICACIÓN MOLECULAR DE LOS ACTINOMICETOS SELECCIONADOS}

El análisis filogenético de las dos cepas seleccionadas muestra alta homología con el género Streptomyces. La cepa señalada como HAA-16 se encuentra muy estrechamente relacionada a Streptomyces albidoflavus con identidades de secuencias de 99 \% (Figura 5), en cambio la cepa HAA-17 sólo fue identificada a nivel de género como Streptomyces sp. Las secuencias del gen 16S ARNr de los actinomicetos seleccionados HAA-16 y HAA-17 fueron depositados en la base de datos del GenBank bajo los códigos de acceso $\mathrm{MH} 746807$ y $\mathrm{MH} 746806$, respectivamente.

\section{DISCUSIÓN}

Estudios similares señalan la presencia de actinomicetos productores de antimicrobianos asociados a hormigas cortadoras de hojas, los cuales poseen una marcada actividad frente a levaduras del género Candida sp.; lo cual es consistente con lo reportado por Barke et al. (22) quienes proponen que los actinomicetos asociados a la tribu Attini, se han desarrollado para promover el tipo correcto de compuestos según la competencia bacteriana y/o antifúngica, proporcionando la combinación adecuada de recursos antimicrobianos. Por tanto, la producción de múltiples compuestos antifúngicos los hace útiles y atractivos para las hormigas como mutualistas.

Existen diversos estudios que confirman la eficacia de los extractos de actinomicetos obtenidos a partir de acetato

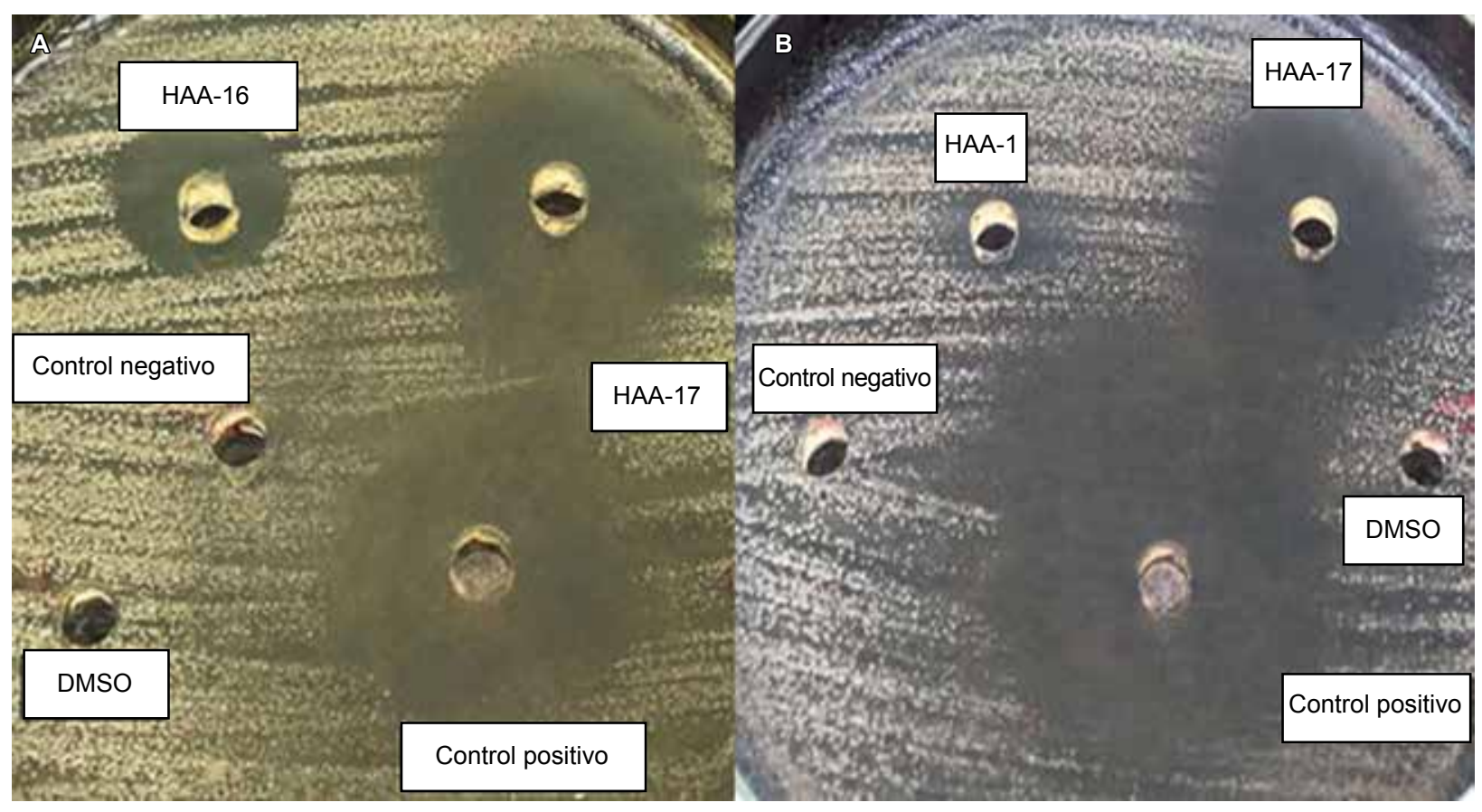

Figura 3. A) Actividad anti-Candida de los extractos orgánicos de acetato de etilo frente a Candida parapsilosis ATCC 7307 y B) Candida albicans ATCC 10231. Control positivo: fluconazol $(20 \mu \mathrm{g} / \mathrm{mL})$. Control negativo: acetato de etilo 


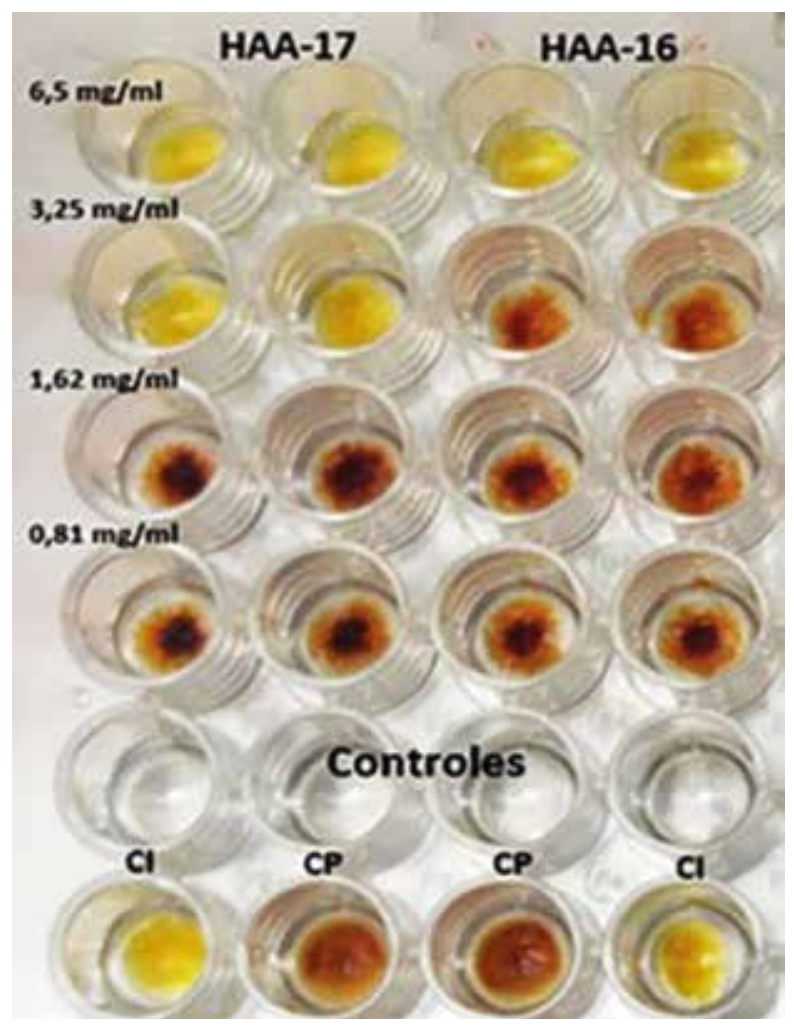

Cl: control de inhibición del patógeno.

$\mathrm{CP}$ : control de crecimiento del patógeno.

Figura 4. Prueba de concentración mínima inhibitoria de los extractos orgánicos obtenidos con acetato de etilo frente a Candida albicans ATCC 7516, usando cloruro de trifenil tetrazolium (TTC) como revelador

de etilo, reportándose una destacada actividad antifúngica frente a $C$. albicans. Mendes et al. ${ }^{(23)}$ determinaron la actividad de extractos de acetato de etilo de actinomicetos asociados a hormigas Trachymyrmex frente a C. albicans, con resultados similares. Asimismo, Narayana et al. (2008) ${ }^{(24)}$ evaluaron el extracto crudo de acetato de etilo de Streptomyces sp, obteniendo máximos resultados de inhibición frente a los microorganismos evaluados en su estudio. Por su parte, Sivasankar et al. ${ }^{(25)}$ ratifican la gran capacidad de los compuestos polares como el acetato de etilo en la recuperación de metabolitos secundarios bioactivos de actinomicetos.

Respecto a la determinación de la CMI, el extracto crudo con acetato de etilo presentó valores entre 3,25-6,50 $\mathrm{mg} \cdot \mathrm{mL}^{-1}$ frente a las distintas cepas del género Candida, siendo estos valores mayores a diferencia de los obtenidos por otros autores, esto debido a que los extractos crudos contienen una mezcla de metabolitos bioactivos que presentan actividad biológica y que en ocasiones generan reacciones antagónicas entre los compuestos bioactivos; por lo tanto, mientras mayor fraccionamiento se realice, mayor será la inhibición (26).
Tal es así que, Mendes et al. (23) determinaron la CMI de las fracciones de los extractos de acetato de etilo frente a especies de Candida con valores que oscilan entre 10$100 \mu \mathrm{g} \cdot \mathrm{mL}^{-1}$. Rashad et al. (27) determinaron la CMI de las fracciones FHM572 y FHM275 del compuesto bioactivo de Streptomyces sp. frente a $C$. albicans, siendo estos valores de 123 y $370 \mu \mathrm{g} \cdot \mathrm{mL}^{-1}$, respectivamente. Asimismo, Narayana et al. (24) determinaron la $\mathrm{CMl}$ de cuatro fracciones del compuesto bioactivo de Streptomyces sp. ANU 6277 frente a $C$. albicans, resultando susceptible a la fracción AF3 a una concentración de $10 \mu \mathrm{g} \cdot \mathrm{mL}^{-1}$.

El análisis filogenético permite asignar a las cepas HAA-16 y HAA-17 como pertenecientes al género Streptomyces; confirmando de esta manera lo señalado en las observaciones morfológicas. Sin embargo, en la cepa HAA-17 no se logró identificarla a nivel de especies; en consecuencia, se plantea estudios moleculares complementarios, tales como: la evaluación de marcadores moleculares específicos o secuencia parcial del gen de la subunidad $\beta$ del ARN polimerasa, pruebas confirmatorias de ácidos grasos, lípidos polares, contenido en guanina y citosina, entre otras ${ }^{(28)}$.

El género Streptomyces representa un modelo en la producción de nuevas y promisorias moléculas antimicrobianas. Se estima que cerca de 10000 metabolitos secundarios bioactivos son producidos por actinomicetos, de ellos, 7600 son producidos por Streptomyces y sólo 150 se han utilizado en áreas como farmacología, agricultura, entre otras ${ }^{(29)}$. En los últimos años se han realizado estudios específicos de metabolitos producidos por actinomicetos asociados a hormigas cortadoras de hojas, obteniendo resultados muy satisfactorios e inclusive llegando a identificar las estructuras de compuestos bioactivos como la candicidina y dentigerumicina purificadas a partir de Streptomyces y Pseudonocardia respectivamente ${ }^{(22,30)}$, los cuales resultaron ser muy activos frente a $C$. albicans.

En la actualidad, los problemas de salud pública por infecciones emergentes provocadas por Candida demandan compuestos eficaces contra este patógeno oportunista. Asimismo, ponen de manifiesto la necesidad de buscar cepas nuevas de actinomicetos bioactivos a partir de fuentes no convencionales. En el presente estudio, a partir de Atta cephalotes se logró aislar un número considerable de estas bacterias con potencial actividad anti-Candida; sin embargo, los estudios realizados sólo se limitaron a escaso número de actinomicetos que fueron finalmente seleccionados para las pruebas cualitativas y cuantitativas de su bioactividad e identificación molecular. Por tratarse de estudios aún muy incipientes limita el planteamiento de posibles aplicaciones en el tratamiento de candidiasis o su uso biotecnológico en salud pública. No obstante, la fortaleza del estudio es que representa un avance preliminar muy interesante en la investigación de compuestos bioactivos que deben ser considerados como punto de partida para futuras investigaciones que incluyan un mayor número de cepas de actinomicetos aislados de Atta cephalotes u otras fuentes no convencionales. 


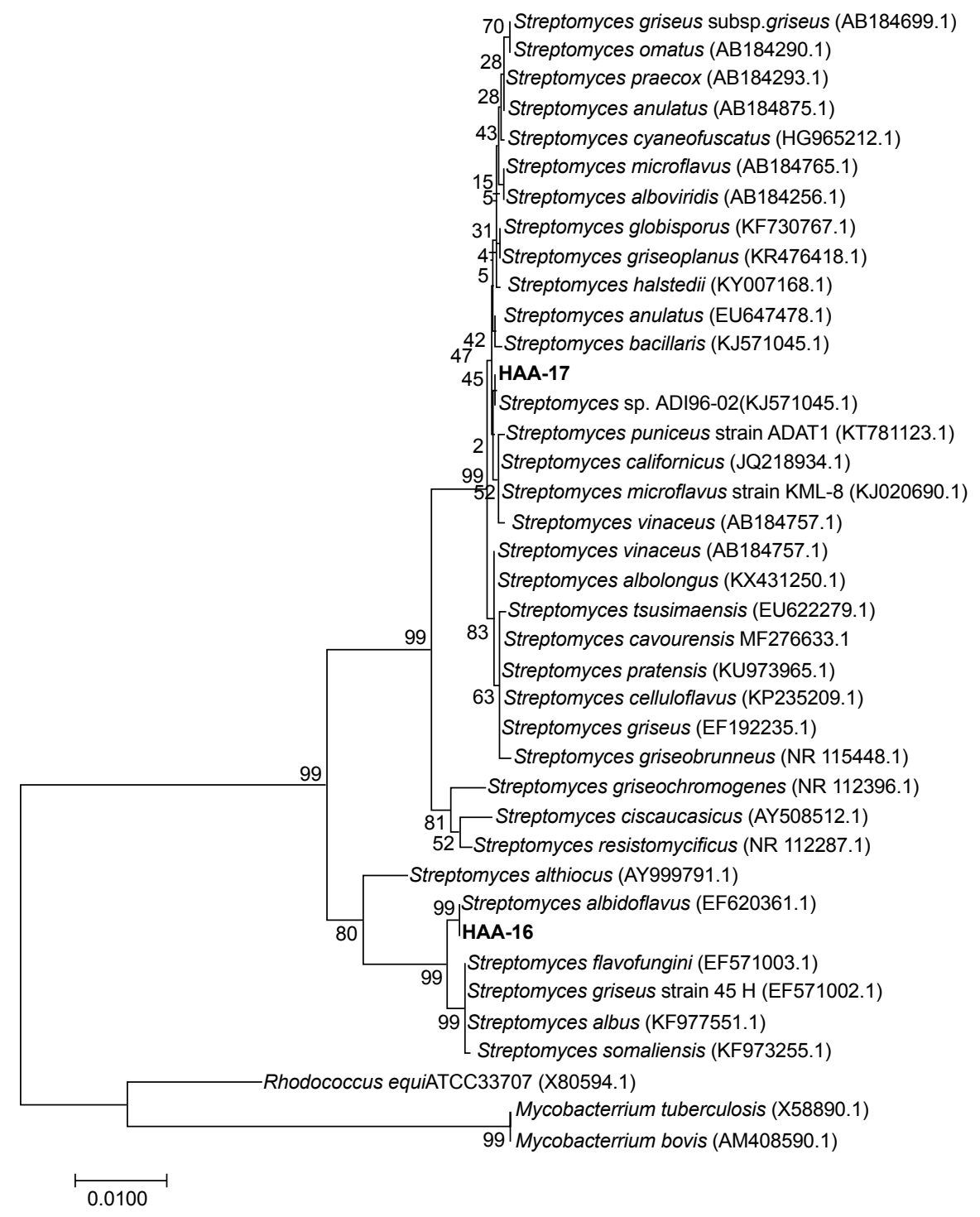

Figura 5. Árbol filogenético de los actinomicetos HAA-16 y HAA-17 aislados de hormigas cortadoras de hojas $A$. cephalotes y especies relacionadas a Streptomyces, en función del análisis del gen ARNr 16S. Las secuencias de Mycobacterium y Rhocococcus fueron elegidas arbitrariamente como grupo externo

Este estudio demuestra que Atta cephalotes es una fuente promisoria de numerosas cepas de actinomicetos con gran capacidad de producir compuestos bioactivos contra las levaduras del género Candida. Esto permite afirmar que representan posibles candidatos para ser utilizados en la lucha contra infecciones y la resistencia microbiana; por consiguiente, podrían tener posibles aplicaciones en el futuro.

Agradecimientos: Al Vicerrectorado de Investigación y Posgrado de la Universidad Nacional Mayorde San Marcos por su financiamiento parcial. A la Blga. Ulrike Tarazona Janampa y a los miembros del laboratorio de Microbiología Molecular y Biotecnología Marina de la Universidad Científica del Sur, por su apoyo en el secuenciamiento de los actinomicetos. Al Mg. Carlos Padilla Rojas, del Laboratorio de Biotecnología y Biología Molecular del Instituto Nacional de Salud y al Blgo. Junior Caro Castro de la Universidad Nacional Mayor de San Marcos por su apoyo en el análisis filogenético. Finalmente, al Blgo. Geancarlos Alarcón de la Universidad Nacional Federico Villareal por su apoyo en la identificación de las hormigas.

Contribuciones de autoría: CAGE ha participado en la concepción y diseño de la investigación, recolección de datos, resultados, análisis, interpretación y redacción del artículo. JLQ ha participado en la dirección del trabajo, revisión crítica del artículo, aprobación de la versión final, aporte de materiales de estudio y obtención del financiamiento (parcial).

Fuentes de financiamiento: El estudio ha sido financiado parcialmente por proyectos internos del Vicerrectorado de Investigación y Posgrado de la Universidad Nacional Mayor de San Marcos, Lima, Perú.

Conflictos de interés: Los autores declaran no tener conflictos de interés. 


\section{REFERENCIAS BIBLIOGRÁFICAS}

1. Lortholary O, Renaudat C, Sitbon K, Madec Y, Denoeud-Ndam L, Wolff M, et al. Worrisome trends in incidence and mortality of candidemia in intensive care units (Paris area, 2002-2010). Intensive Care Med. 2014; 40(9):1303-12. doi: 10.1007/s00134-014-3408-3.

2. Limia $O$, Betancourt A, Lesteiro $M$, Faure R. Prevalencia por diagnóstico inmunológico de Candida spp, Trichomonas vaginalis y Gardnerella vaginalis en mujeres embarazadas a nivel primario del sistema de salud. Rev Cubana Obstet Ginecol. 2010;36(1):65-72.

3. Torres N. Métodos para la detección de resistencia a los antimicóticos. Infectio. 2005;9:188-96.

4. Busti E, Monciardini P, Cavaletti L, Bamonte R, Lazzarini A, Sosio M, et al. Antibiotic-producing ability by representatives of a newly discovered lineage of actinomycetes. Microbiology. 2006;152(3):675-83. doi: 10.1099/mic.0.28335-0.

5. Huang W, Beharry Z, Zhang Z, Palzkill T. A broad-spectrum peptide inhibitor of lactamase identified using phage display and peptide arrays. Protein Eng. 2003;16(11):853-60. doi: 10.1093/protein/gzg108.

6. Ventura M, Canchaya C, Tauch A, Chandra G, Fitzgerald G, Chater K, et al. Genomics of actinobacteria: Tracing the evolutionary history of an ancient phylum. Microbiol Mol Biol Rev. 2007:71(3):495548. doi: 10.1128/MMBR.00005-07.

7. Procópio R, Silva I, Martins M, Azevedo J, Araújo J. Antibiotics produced by Streptomyces. Braz J Infect Dis. 2012;16(5):46671. doi: 10.1016/j.bjid.2012.08.014.

8. Goodfellow M, Fiedler H. A guide to successful bioprospecting: Informed by actinobacterial systematics. Antonie Van Leeuwenhoek. 2010;98(2):119-42. doi: 10.1007/s10482-010-9460-2.

9. Abidin Z, Malek N, Zainuddin Z, Chowdhury A. Selective isolation and antagonistic activity of actinomycetes from mangrove forest of Pahang, Malaysia. Front Life Sci. 2016;9(1):24-31. doi: 10.1080/21553769.2015.1051244.

10. Seipke R, Kaltenpoth M, Hutchings M. Streptomyces as symbionts: an emerging and widespread theme? FEMS Microbiol Rev. 2012;36:862-76. doi: 10.1111/ j.1574-6976.2011.00313.x.

11. Behie S, Bonet B, Zacharia V, McClung D, Traxler M. Molecules to ecosystems: Actinomycete natural products in situ. Front Microbiol. 2016;7:2149. doi: 10.3389/fmicb.2016.02149.
12. Currie C, Scott J, Summerbell R, Malloch $\mathrm{D}$. Fungus-growing ants use antibiotic-producing bacteria to control garden parasites. Nature. 1999;398:701-4. doi: 10.1038/19519.

13. Paillié D. Aislamiento y caracterización preliminar de genes de policétido sintasas tipo I en actinomicetos aislados a partir de hormigas cortadoras de hojas Atta cephalotes [Tesis de licenciatura]. Bucaramanga: Universidad Industrial de Santander; 2011 [citado el 15 de mayo de 2018]. Disponible en: http://tangara.uis.edu.co/ biblioweb/tesis/2011/142134.pdf

14. Kokare CR, Mahadik KR, Kadam SS, Chopade BA. Isolation of bioactive marine actinomycetes from sediments isolated from Goa and Maharashtra coastlines (west coast of India). Indian J Mar Sc. 2004; 33:248-56. doi: 10.3923/ pjbs.2006.470.472.

15. León J, Liza L, Soto I, Cuadra DL, Patiño L, Zerpa R. Actinomycetes bioactivos de sedimento marino de la costa central del Perú. Rev Per Biol. 2007; 14(2):259-70. doi:http://dx.doi.org/10.15381/rpb. v14i2.1796.

16. Aponte J, León J, Rojas R, Montero S, Loayza L. Actividad antimicrobiana y sinérgica de metabolitos producidos por Streptomyces erythrogriseus M10-77 de origen marino. Rev Soc Ven Microbiol. 2015; 33:13-19.

17. Liu C, Westley J, Herman T, Prosser B, Palleroni N, Evans R, et al. Novel polyether antibiotics X-14873A, G and H produced by Streptomyces: Taxonomy of the producing culture, fermentation, biological and ionophorous properties of the antibiotics. J Antibiot. 1986;39(12):1712-18. doi: 10.7164/antibiotics.39.1712.

18. Pandey B, Ghimire P, Agrawal VP. Studies on the antibacterial activity of the actinomycetes isolated from the Khumbu Region of Nepal. J Biol Sci. 2004;23:44-53.

19. Karthy E, Ranjitha P, Mohankumar A. Antimicrobial potential of plant seed extracts against Multidrug Resistant Methicillin Resistant Staphylococcus aureus (MDRMRSA). Int J Biol. 2009;1(1):3440. doi: 10.5539/ijb. v1n1p34.

20. Altschul SF, Gish W, Miller W, Myers EW, Lipman DJ. Basic Local Alignment Search Tool. J Mol Biol. 1990; 215(3):403-10. doi: 10.1016/S0022-2836(05)80360-2.

21. Kimura M. A simple method for estimating evolutionary rates of base substitutions through comparative studies of nucleotide sequences. J Mol Evol. 1980;16(2):11120. doi: 10.1007/BF01731581.
22. Barke J, Seipke RF, Gruschow S, Heavens D, Drou N, Bibb M, et al. A mixed community of actinomycetes produce multiple antibiotics for the fungus farming ant Acromyrmex octospinosus. BMC Biol. 2010; 8(1):109. doi: 10.1186/17417007-8-109.

23. Mendes T, Borges W, Rodrigues A, SolomonS, Vieira P, Duarte M, et al. Anti-Candida properties of Urauchimycins from actinobacteria associated with Trachymyrmex ants. BioMed Res Int. 2013;9:1-8. doi: 10.1155/2013/835081.

24. Narayana K, Prabhakar P, Vijayalakshmi M, Venkateswarlu Y, Krishna P. Study on bioactive compounds from Streptomyces sp. ANU 6277. Pol J Microbiol. 2008;57(1):35-9.

25. Sivasankar P, Manivasagan P, Vijayanand P, Sivakumar K, Sugesh S, Poongodi S, et al. Antibacterial and brine shrimp lethality effect of marine actinobacterium Streptomyces sp. CAS72 against human pathogenic bacteria. Asian Pac J Trop Dis. 2013;3(4):286-93. doi: 10.1016/S22221808(13)60071-7.

26. De Silvestre J, Zea S, Duque C. Actividad antibacteriana de algunas esponjas del Caribe Colombiano. Rev Colomb Cienc Quim Farm. 1994;22:21-6.

27. Rashad F, El-Nasser N, Dawoud I, Motawe F. Isolation and characterization of antibiotic/ antitumor producing Streptomyces. Res J Pharm Biol Chem Sci. 2015;6(2):1917-29.

28. Kim BJ, Kim CJ, Chun J, Koh YH, Lee $\mathrm{SH}$, Hyun JW, et al. Phylogenetic analysis of the genera Streptomyces and Kitasatospora based on partial RNA polymerase $\beta$-subunit gene (rpoB) sequences. Int $\mathrm{J}$ Syst Evol Microbiol. 2004; 54(2):593-8. doi: 10.1099/ijs.0.02941-0.

29. Olano C, Lombó F, Méndez C, Salas J. Improving production of bioactive secondary metabolites in actinomycetes by metabolic engineering. Metab Eng. 2008; 10(5):281-92. doi: 10.1016/j.ymben.2008.07.001.

30. Oh DC, Poulsen M, Currie C, Clardy J. Dentigerumycin: a bacterial mediator of an ant-fungus symbiosis. Nat Chem Biol. 2009; 5(6):391-3. doi: 10.1038/nchembio.159.

Correspondencia: Jorge León Quispe. Dirección: Facultad de Ciencias Biológicas, Universidad Nacional Mayor de San Marcos, Lima, Perú.

Teléfono: 01-6197000 anexo 1542.

Correoelectrónico: jleonq@unmsm.edu.pe 